\title{
Acute renal failure following Yersinia pseudotuberculosis septicaemia
}

\author{
A. Davenport, B. O'Connor and R. Finn \\ Royal Liverpool Hospital, Prescot Street, Liverpool L1 8XP, UK.
}

\begin{abstract}
Summary: Although Yersinia pseudotuberculosis has not been previously reported to cause acute renal failure, it accounted for two cases reported here out of eighty referrals with acute renal failure to our renal unit over the last year. This may suggest that the incidence of Yersinia pseudotuberculosis may be greater than that previously reported, and it should be suspected in patients presenting with bloody diarrhoea in association with renal failure. The septicaemic form of the disease is life threatening and requires intensive supportive therapy.
\end{abstract}

\section{Introduction}

Yersinia pseudotuberculosis, a Gram negative coccobacillus with a world wide distribution, ${ }^{1}$ usually causes an acute mesenteric lymphadenitis simulating acute or subacute appendicitis. Occasionally a severe septicaemic illness similar to typhoid may develop, usually in patients who are iron overloaded. ${ }^{2}$

$Y$. pseudotuberculosis has not previously been reported to cause acute renal failure. We report two cases of severe infection complicated by acute renal failure. Neither patient was iron overloaded.

\section{Case reports}

Case 1

A 68 year old woman presented with a 3-day history of colicky lower abdominal pain associated with bloody diarrhoea. Initial management was with intravenous fluids, metronidazole and cefuroxime. The serum amylase was elevated at 1,414 IU/1 (normal < 200 IU/ 1). Her condition deteriorated over the next 6 days as she developed both disseminated intravascular coagulopathy (DIC) and oliguric renal failure. Her haemoglobin had fallen from $11.5 \mathrm{~g} / \mathrm{dl}$ to $4.5 \mathrm{~g} / \mathrm{dl}$, the platelet count $225 \times 10^{9} / 1$ to $45 \times 10^{9} / 1$ but the white blood cell count remained stable around $8.4 \times 10^{9} / 1$ with a predominant neutrophilia. Fibrin degradation products were present in the serum at a titre of $1 / 160$ and the prothrombin time was elongated at 24 seconds (control 12). The serum urea and creatinine had risen from $14.5 \mathrm{mmol} / 1$ and $156 \mu \mathrm{mol} / 1$ to $37.5 \mathrm{mmol} / \mathrm{l}$ and

Correspondence: A. Davenport M.A., M.R.C.P.

Accepted: 6 March 1987
$1057 \mu \mathrm{mol} / 1$ respectively. Transfer to the regional renal unit for haemodialysis was arranged. On arrival she was pyrexial, temperature $38^{\circ} \mathrm{C}$, pale with a sinus tachycardia and a blood pressure of $130 / 70 \mathrm{mmHg}$. She was tender in the right hypochondrium. Sigmoidoscopy revealed hyperaemia of the rectal mucosa. She required daily haemodialysis and then ventilation when adult respiratory distress syndrome (ARDS) developed. The abdomen became distended and tender and a laparotomy was performed. The small bowel was congested, but there was no evidence of ulceration. A renal biopsy showed marked acute tubular necrosis with widespread intra-renal thrombosis.

Slowly, her condition improved and after 3 weeks, haemodialysis was no longer required. She was discharged home 40 days after admission to hospital with a serum creatinine of $284 \mu \mathrm{mol} / \mathrm{l}$, which continued to fall on clinic review over the next 4 months. All cultures of blood, urine and stool were negative. In view of the history of bloody diarrhoea and DIC, serology for $Y$. pseudotuberculosis was requested. The titre to $Y$. pseudotuberculosis type IV rose significantly from zero to $1 / 1,280$ over the 6 weeks of her illness. She was not iron overloaded as evidenced by low serum iron and normal ferritin levels.

\section{Case 2}

Within a week of admission of the first patient, an 18 year old man was admitted with an 8 day history of colicky lower abdominal pain also associated with bloody diarrhoea. He was pyrexial, temperature $38^{\circ} \mathrm{C}$, pale and dehydrated with a distended tympanitic abdomen. Investigations confirmed a DIC with acute

(C) The Fellowship of Postgraduate Medicine, 1987 
renal failure, haemoglobin was $4.9 \mathrm{~g} / \mathrm{dl}$ with a leucocytosis of $28.5 \times 10^{9} / 1$ with a predominant neutrophilia and a platelet count of $49 \times 10^{9} / 1$. The prothrombin time was prolonged at 26 seconds (control 12) and the fibrin degradation products elevated at a titre of $1 / 80$. The serum urea was elevated at $80 \mathrm{mmol} / \mathrm{l}$ and the creatinine at $831 \mu \mathrm{mol} / \mathrm{l}$. Plain abdominal X-rays showed a grossly dilated large bowel; sigmoidoscopy and single contrast barium enema did not show any major mucosal abnormality. He was transfused with blood and fresh frozen plasma, treated with piperacillin, tobramycin, metronidazole and haemodialysed. His respiratory function deteriorated and he required ventilation. A renal biopsy revealed acute tubular necrosis with widespread intra-renal thrombosis. His condition improved and after 4 days, ventilation was discontinued, as was haemodialysis as he developed polyuria. Seventeen days after admission, he was discharged home with a normal serum creatinine. All cultures of blood, urine and stool were negative. Yersinia serology was requested in view of the clinical presentation, the titre to $Y$. pseudotuberculosis type IV rose significantly from zero to $1 / 620$ over the three weeks of

\section{References}

1. Anonymous, Yersiniosis today (Editorial). Lancet 1984, i: 84-85.

2. Swaminathan, M.C., Harmon, M.C. \& Metilman, I.S. A Review. Yersinia enterocolitica. J Appl Bacteriol 1982, 52: $151-183$.

3. Chiu, H.Y., Flynn, D.M., Hoffbrand, A.V. \& Politis, D. Infection with Yersinia enterocolitica in patients with iron overload. Br Med J 1986, 292: 96. his illness. Both the serum iron and ferritin were reduced on admission.

\section{Discussion}

We have reported two cases of acute renal failure due to infection with Yersinia pseudotuberculosis type IV. Both presented with bloody diarrhoea and then developed a generalized DIC with extensive intrarenal thrombosis resulting in acute renal failure. Adult respiratory distress syndrome supervened in both cases.

The septicaemic form of yersinia infection has been reported to have a high mortality $(70 \%)$ despite antibiotics and is commoner in patients who are iron overloaded or have liver disease. ${ }^{3}$

Yersinia has been reported to cause both diffuse proliferative ${ }^{4}$ and membranoproliferative glomerulonephritis. ${ }^{5}$ However, there was no primary glomerular pathology in either of our biopsies, the main pathology being extensive intra-renal thrombosis with acute tubular necrosis.
4. Awunor - Renner, C. \& Lawande, R.V. Yersinia an chronic glomerulopathy in the Savannah region Nigeria. Br Med J 1982, 285: 1464.

5. Denneberg, T., Friedberg, M., Samuelson, T. \& Winblad, S. Glomerulonephritis in infections with Yersinia enterocolitca O-stereotype 3.1. Evidence for glomerular involvement in acute cases of yersinosis. Acta Med Scand 1981, 209: 97-101. 